\title{
An Efficient Technique for Image Retrieval from the Large Database on the Basis of Color and Texture
}

\author{
Mayank Jain \\ Student of M.Tech VI semester CSE \\ University Institute of Technology \\ Barkatullah University, \\ Bhopal, M.P.,India.
}

\author{
Divakar Singh \\ Assistant Professor, CSE \\ University Institute of Technology \\ Barkatullah University, \\ Bhopal, M.P.,India.
}

\begin{abstract}
Now a day's development of multimedia technology, the possibilities of utility of large databases is rapidly increasing. To handle its management and retrieval CBIR is the best and effective method. CBIR technique uses the visual contents like as color, shape and texture that are called features, to searching, browsing, and navigation of query images for large image databases. Color is the visual perceptual property corresponding in humans to the categories called red, blue and yellow etc. Texture is the image and especially physical quality of a surface. Texture is the characteristic structure of the interwoven or intertwined outfit, strands or the like that make up a textile fabric. In this paper we present utility of CBIR system with color and texture features. And we design a color filter with the help of extract the red channel, green channel and blue channel from the original image. After it we find a texture of all channels with the help of statistical method. The combination of texture of red channel, green channel and blue channel we create a feature vector for all images of the database. Experimental results are shows the average accuracy, average precision rate and average retrieval rate. That is better than other existing method.
\end{abstract}

\section{Keywords}

CBIR, feature vector, Euclidean distance, precision and recall.

\section{INTRODUCTION}

The image retrieval system works as a classifier to break up the images in the image database into two modules, either relevant or irrelevant. When results are irrelevant, the feedback loop is repeated until the user is satisfied. Relevance feedback involves the user to label the similar and dissimilar image. An efficient image retrieval technique is used to retrieve similar images in various stages. The images firstly retrieved on color basis and the resultant retrieved images further match with their shape and texture feature respectively. Content-based image retrieval (CBIR) is the use of computer vision to the image retrieval difficulty that is the crisis of searching for digital images in huge databases. "Content-based" means that the search will evaluate the actual contents of the image. 'Content' word refers colors, shapes, textures, or some other information that can be taking from the image itself. The CBIR technique is used in various fields such as medical, agriculture, security, weather forecasting, biological modeling, remote sensing, architecture, web image classification, crime prevention, satellite images, identification and retrieval, etc.[1][2]

CBIR consists the two phases first is the indexing phase where image information like the color, shape, and texture is enumerated into features that are stored in an index data structure. Second is the retrieval phase where is searching for an image in the CBIR index. Color similarity is obtained by computing a color histogram for every image with the purpose of identifies the ratio of pixels within an image holding specific values. Tentative images based on the colors they contain are one of the most commonly used techniques because it does not depend on image size or direction. The color searches will usually keep comparing color histograms. Texture descriptor provides a measure of properties such as smoothness, roughness, and regularity. The texture of the region is structural, statistical and spectral are three principal approaches used in image processing. Shape is the external form, contours or outline of someone or something. A shape is the form of an object or its external surface as opposed to other properties such as color, texture or material composition.

\section{LITERATURE SURVEY}

Bugatti et al. (2014) present a novel approach to perform similarity queries over medical images, maintaining the semantics of a given query posted by the user. And also present a highly effective strategy to survey user profiles, taking advantage of such labeling to implicitly gather the user perceptual similarity. Experiments on medical images show that the method is effective and can improve the decision making process during analysis. [5]

Verma et al. (2015) present a new image retrieval technique; local extrema co-occurrence patterns (LECoP) using the HSV color space. HSV color space is used in this method to utilize the color, intensity and brightness of images. Local extrema patterns are applied to define the local information of image, and gray level co-occurrence matrix is used to obtain the cooccurrence of LEP map pixels. The presented method is tested on five standard databases called Corel, MIT VisTex and STex, in which Corel database includes Corel-1k, Corer-5k and Corel-10k databases. Also, this algorithm is compared with previous proposed methods, and results in terms of precision and recall. [6]

Meng jian et al. (2015) presents a novel interactive image retrieval framework using constraints. First, extract the user region of interest (ROI) from queries by simple user interaction using adaptive constraints-based seed propagation (ACSP), and obtain initial retrieval results based on the ROI. Then, improve the retrieval results by active learning from the user relevance feedback using ACSP. Since ACSP is very effective in propagating the user interactive information of constraints by employing a kernel learning strategy, it successfully learns the correlation between low-level image features and high-level semantics from the ROI and relevance feedbacks. Experimental results demonstrate that the proposed 
framework remarkably improves the image retrieval performance by ACSP-based constraint propagation in terms of both effectiveness and efficiency. [7]

Khemchandani et al. (2015) present Ternary Decision Structure based multi-category twin support vector machines (TDS-TWSVM) classifier. Twin support vector machines (TWSVM) formulation deals with finding non-parallel plane classifier which is obtained by solving two related Quadratic Programming Problems (QPPs). The proposed TDS-TWSVM classifier is an extension of TWSVM so as to handle multiclass data and is more efficient in terms of training and testing time of classifiers. The experimental results depict that TDSTWSVM outperforms One-Against-All TWSVM (OAATWSVM) and binary tree-based TWSVM (TB-TWSVM) in terms of classification accuracy. [8]

Bhaskar reddy et al. (2014) integrates the concept of directional local extremas and their magnitude based patterns for content based image indexing and retrieval. The standard directional local extrama pattern (DLEP) extracts the directional edge information based on local extrema in $0^{\circ}$, $45^{\circ}, 90^{\circ}$, and $135^{\circ}$ directions in an image. However, they are not considering the magnitudes of local extremas. The proposed method integrates these two concepts for better retrieval performance. The performance of the proposed method is compared with DLEP, local binary patterns (LBPs), block-based LBP (BLK_LBP), center-symmetric local binary pattern (CS-LBP), local edge patterns for segmentation (LEPSEG) and local edge patterns for image retrieval (LEPINV) methods by conducting two experiments on benchmark databases, viz. Corel-5K and Corel-10K databases. The results after being investigated show a significant improvement in terms of their evaluation measures as compared to other existing methods on respective databases. [9]

Yasmin et al. (2014) present in our paper, images are decomposed in equal squares of minimum $24 \times 16$ size and then edge detection is applied to those decomposed parts. Pixels classification is done on the basis of edge pixels and inner pixels. Features are selected from edge pixels for populating the database. Moreover, color differences are used to cluster same color retrieved results. Precision and recall rates have been used as quantification measures. It can be seen from the results that proposed method shows a very good balance of precision and recall in minimum retrieval time, achieved results are comprised of $66 \%-100 \%$ rate for precision and $68 \%-80 \%$ for recall. [10]

Zarchi et al. (2014) proposed A semantic model for general purpose content-based image retrieval systems. This model is used to overcome semantic gap in CBIR. In this model an interactive image segmentation algorithm is carried out on the query image to extract the user-interested regions. To recognize the image objects from regions, a neural network classifier is used in this model. The ant colony optimization algorithm is employed to eliminate irrelevant and redundant features. To find the most similar images to the query image, the similarity between images is measured based on their semantic objects which are defined according to a predefined ontology. [11]

Raghuwanshi et al. (2016) proposed Texture image retrieval using adaptive tetrolet transforms. Tetrolets provide fine texture information due to its different way of analysis. Tetrominoes are applied at each decomposition level of an image and best combination of tetrominoes is selected, which better shows the geometry of an image at each level. All three high pass components of the decomposed image at each level are used as input values for feature extraction. A feature vector is created by taking standard deviation in combination with energy at each subband. Retrieval performance in terms of accuracy is tested on group of texture images taken from benchmark databases: Brodatz and VisTex. Experimental results indicate that the proposed method achieves $78.80 \%$ retrieval accuracy on group of texture images D1 (taken from Brodatz), $84.41 \%$ on group D2 (taken from VisTex) and $77.41 \%$ on rotated texture image group D3 (rotated images from Brodatz). [12]

Vipparthi et al. (2015) proposed Dual directional multi-motif XOR patterns: A new feature descriptor for image indexing and retrieval. This method is entirely different from the existing motif representation. The performance of the proposed method is tested by conducting two experiments on Corel-5000 and Corel-10,000 databases. Experimental results demonstrate that it is much more efficient in terms of average retrieval precision (ARP) and average retrieval rate (ARR). [13]

Yang et al. (2015) analyze the rotation, scaling, and translation (RST) invariant property of EMs, and propose a content-based image retrieval approach using invariant EMs. Experimental results show that the EMs can be used as an effective descriptor of global image content, and the proposed retrieval approach yields higher retrieval accuracy than some current state-of-the-art retrieval methods. [14]

\section{PROPOSED METHODOLOGY}

\section{Algorithm}

Input: Query Image Output: Retrieve similar images.

Step 1: Load query image.

Step 2: Perform the preprocessing on the query image.

Step 3: Apply the proposed color filter on query image to extract the red channel, green channel and blue channel.

Step 4: After extracting the channel we convert these channels into gray level images and perform the texture analysis on each channel with the help of statistical approach.

Step 5: Now we combining the texture properties of all channel and obtain a feature vector.

Step 6: We apply distance measure to compare the query image with the database images. The feature vector of all the images are also computed in the same manner and stored in the feature database.

Step 7: Retrieve the database images having best matches with the query image.

Step 8: Perform the majority voting to find the category of which maximum number of images are retrieved.

Step 9: Assign the type of category to the query image.

The figure 3.1 is show the working of proposed method CBIR using color and texture features.

\section{FEATURE VECTOR PREPARATION}

Images to be stored in database are taken one by one and their color and texture features are calculated. For each image color feature is extracted using analysis of the pixels. After then on the basis of pixels we get the red channel, green channel and blue channel of the image. To get the texture information, we apply statistical texture approach on the each channel on the 
basis of average gray level, average contrast, smoothness, third moment, uniformity, and entropy.

All channels of the texture feature vectors are combined in to form a single feature vector which is indexed in the database using primary key as index.
The calculated feature vector is stored in the relational database table using primary key as sequential counter (see Table. No.4.1), the initial value of the counter is set as 1 . The value of counter is increased by one automatically, when an image feature vector is stored into the database. The name of images in the database are unique i.e. no two images in the database can have the same name.

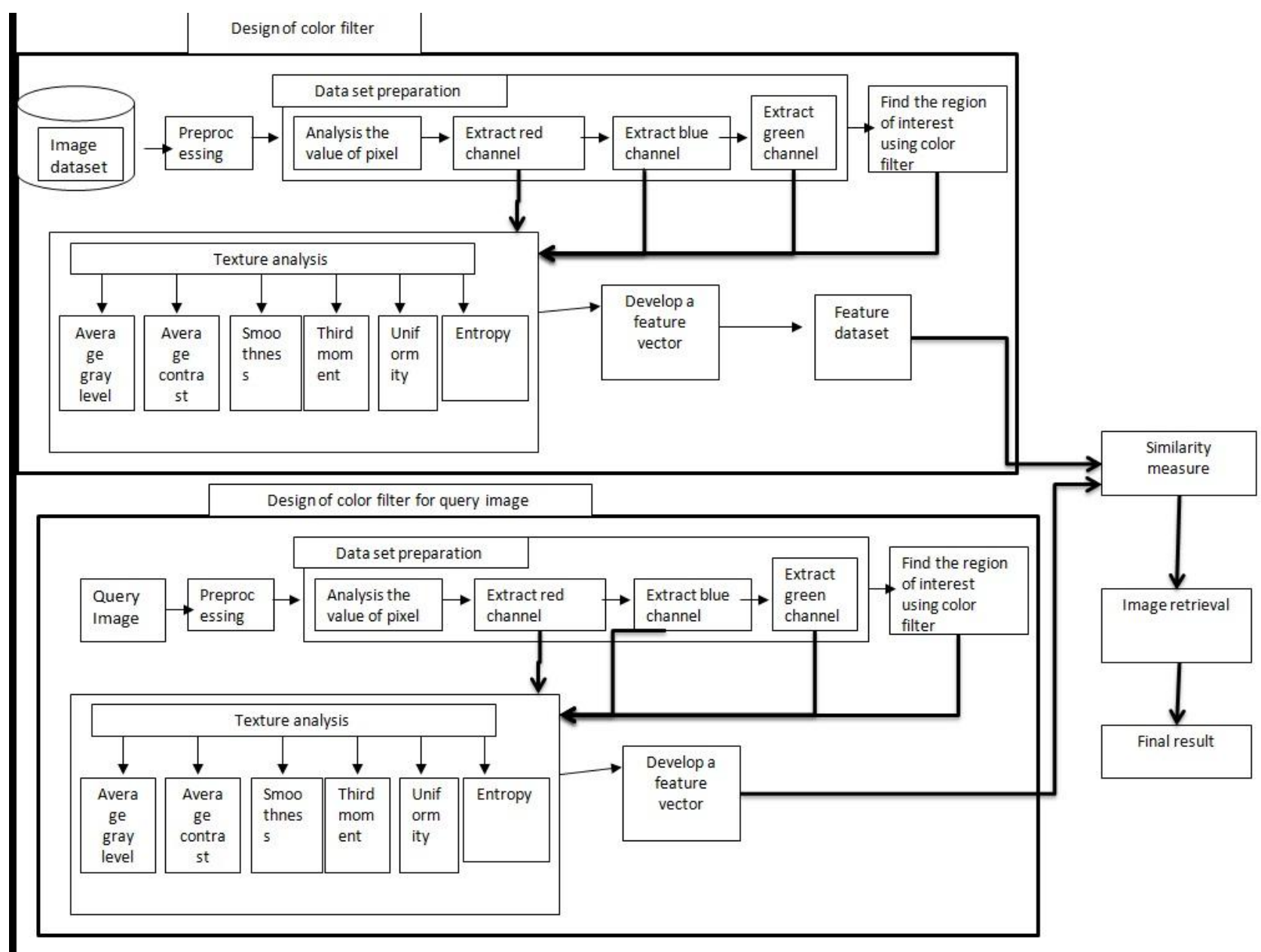

Fig. 3.1: Proposed method CBIR using color and texture features.

Table No. 4.1: Structure of feature vector

\begin{tabular}{|c|c|c|c|c|c|c|c|c|c|c|c|c|c|c|c|c|c|c|c|}
\hline \multirow{6}{*}{ Index } & \multicolumn{18}{|c|}{ Color feature } & \multirow{6}{*}{ Image name } \\
\hline & \multicolumn{6}{|c|}{ Red channel (R) } & \multicolumn{6}{|c|}{ Green channel $(\mathrm{G})$} & \multicolumn{6}{|c|}{ Blue channel (B) } & \\
\hline & \multicolumn{18}{|c|}{ Apply Texture feature on each channel } & \\
\hline & $\mathrm{R}$ & $\mathrm{R}$ & $\mathrm{R}$ & $\mathrm{R}$ & $\mathrm{R}$ & $\mathrm{R}$ & G & $\mathrm{G}$ & $\mathrm{G}$ & $\mathrm{G}$ & $\mathrm{G}$ & $\mathrm{G}$ & $\mathrm{B}$ & B & B & B & $\mathrm{B}$ & $\mathrm{B}$ & \\
\hline & $\mathrm{T}$ & $\mathrm{T}$ & $\mathrm{T}$ & $\mathrm{T}$ & $\mathrm{T}$ & $\mathrm{T}$ & $\mathrm{T}$ & $\mathrm{T}$ & $\mathrm{T}$ & $\mathrm{T}$ & $\mathrm{T}$ & $\mathrm{T}$ & $\mathrm{T}$ & $\mathrm{T}$ & $\mathrm{T}$ & $\mathrm{T}$ & $\mathrm{T}$ & $\mathrm{T}$ & \\
\hline & 1 & 2 & 3 & 4 & 5 & 6 & 1 & 2 & 3 & 4 & 5 & 6 & 1 & 2 & 3 & 4 & 5 & 6 & \\
\hline 1 & . & . & . & . & . & ... & $\ldots$ & $\cdot$ & . & . & . & . & . & . & $\ldots$ & . & $\ldots$ & $\ldots$ & 1.jpg \\
\hline 2 & . & - & . & . & . & . & . &. & . & . & . & . & . & . & . & $\ldots$ & . & . & .. \\
\hline 3 & & & & & & & & & & & & & & & & & & & .. \\
\hline$\ldots$ & & & & & & & & & & & & & & & & & & & .. \\
\hline$\ldots$ & & & & & & & & & & & & & & & & & & & .. \\
\hline$\ldots$ & & & & & & & & & & & & & & & & & & & .. \\
\hline $\mathrm{N}$ & & & & & & & & & & & & & & & & & & & $\ldots$ \\
\hline
\end{tabular}




\section{DISTANCE MEASURE}

Distance measures are used for comparing the similarity of two images. There are different kinds of similarity measurements like Euclidean distance, histogram intersection, Bhattacharya distance and Mahalanobis distance for CBIR applications.

\subsection{Euclidean Distance}

Let $F_{Q}[i]$ be the feature vector of query image and $F_{D B}[i]$ be the feature vector of database image and The Euclidean distance between the query image and the target image is given by equation

$$
\mathrm{D}=\sqrt{\left(F_{Q}[i]-F_{D B}[i]\right)^{2}}
$$

In Euclidean distance, the least value of distance measure indicates the similarity. [4]

\section{SIMILARITY MEASURE \\ 6.1 Wavelet Decomposed Color Histogram (WDH)}

This wavelet transform identifies the core pixels of the image that make up the image at different levels. Each level gets the wavelet and the image gets on condensing to get the actual pixels of the image out. Perform Simple wavelet decomposition [15], [16] on the image and now consider each pass as a vector value. This brings the wavelet vector.

Feature Extraction using Wavelet Decomposition:

- Convert all data base images into gray images.

- Decompose each image in the Multi wavelet domain

- Compute the standard deviation on each sub band of the Multi Wavelet decomposed image.

- $\quad$ Each sub band standard deviation forms a resulting feature vector.

- Store this feature vector in database its act like one of the feature.

\subsection{Color coherence vector $(\mathrm{CCV})$}

Color coherence vector is proposed by Pass et al. (1997) to compare the images with the help of the density of the color. Color coherence vector first quantize the RGB color channel to reduce the size of the resulting descriptor and to avoid the differences caused by small changes between surrounding pixels. After quantization the method computes the connected components and classifies these connected components either coherent or incoherent. A connected component having a particular color is coherent if its pixels are the member of some sizable contiguous region of that color and these pixels are classifies as coherent pixels. Two color histogram of 32bins for coherent and incoherent pixels are computed to characterize the image pixels and finally concatenated to produce the single feature descriptor of 64 - dimensions.[4]

\section{PERFORMANCE EVALUATION}

The performance of retrieval of the system can be measured in terms of its recall and precision. Recall measures the ability of the system to retrieve all the models that are relevant, while precision measures the ability of the system to retrieve only the models that are relevant. They are defined as:
Precision $=$ No. of relevant images retrieved $/$ Total no. of images retrieved.

Recall $=$ No. of relevant image retrieved $/$ Total no. of relevant images in the database.

The average accuracy is defined as follow:

Average accuracy $=\frac{1}{n} \sum_{i=1}^{n} a(X i)$

Where $\mathrm{n}$ is the total number of images in the dataset and $a(X i)$ is the accuracy for test images (Xi) and defined as

$a(X i)=\left\{\begin{array}{l}100, \text { if } \mathrm{Xi} \text { is classified correctly } \\ 0, \text { else }\end{array}\right.$

\section{EXPERIMENTAL RESULTS}

This section provides the experimental evaluation of present method. A computer system having Pentium IV, $2.8 \mathrm{GHz}$ processor and 2 GB RAM is used for conducting experiments. This system has been implemented in MATLAB.

The performance of the proposed image retrieval system is tested using Corel database downloaded from http://wang.ist.psu.edu/docs/related/. The Corel image dataset contains 1000 images having 100 images of 10 categories, which include African people, Beaches, Building, Buses, Dinosaurs, Elephant, Flower, Horses, Mountains, Foods, respectively. Example images from each of these categories are shown in figure 8.1. Our experiments are performing on the dataset of 500 images that are taken from the corel-1000 images dataset. Experimental results show the average recall rate, average precision rate and average accuracy. All the results shown in fig 8.2 to 8.4 .
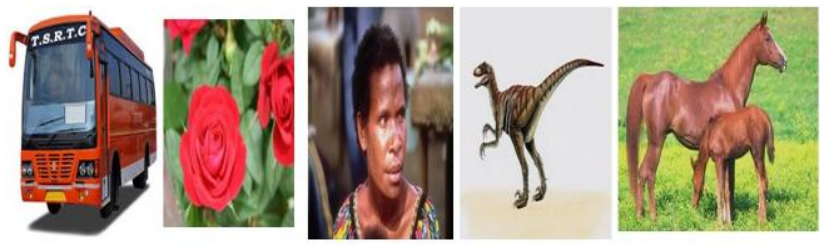

Fig. 8.1: Sample images from each category of the dataset.

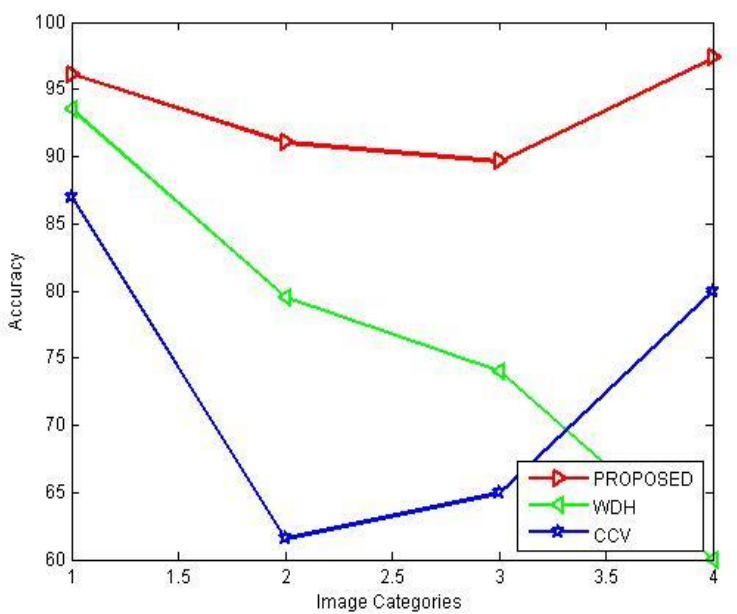

Fig. 8.2: Average accuracy of each class of images. 
The figure 8.2 show the average accuracy for each class of the dataset and compare the proposed method with the WDH and $\mathrm{CCV}$ similarity measures. That provides the better results at each categories of the dataset.

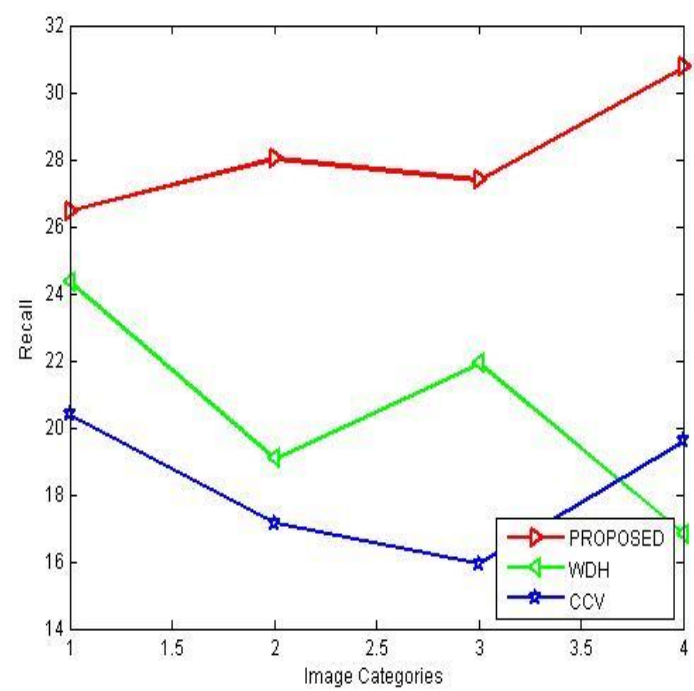

Fig. 8.3: Average recall rate of each class of images.

The figure 8.3 show the average recall for each class of the dataset and compare the proposed method with the WDH and $\mathrm{CCV}$ similarity measures. That provides the better results at each categories of the dataset.

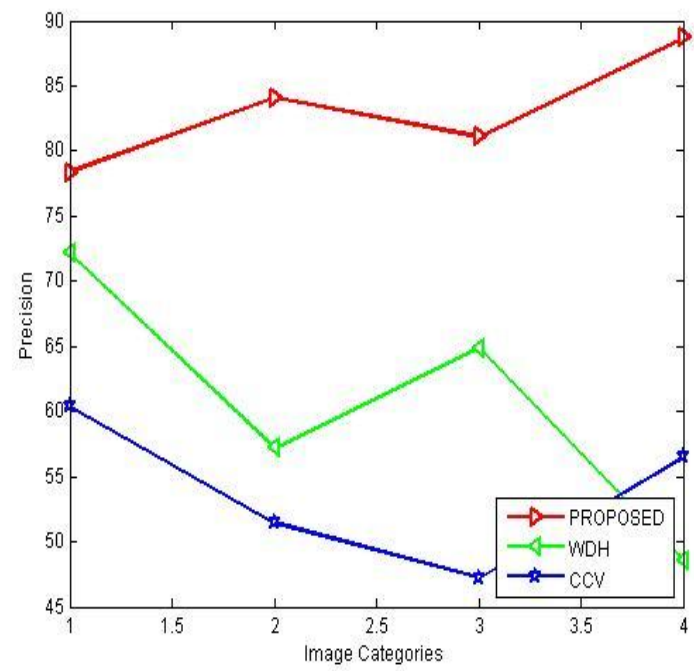

Fig. 8.4: Average precision rate of each class of images.

The figure 8.4 show the average precision for each class of the dataset and compare the proposed method with the WDH and $\mathrm{CCV}$ similarity measures. That provides the better results at each categories of the dataset.

\section{CONCLUSION}

In this paper we show the advantages of CBIR system. And we design a color filter with the help of it we extract the red channel, green channel and blue channel from the original image. After it we find a texture of all channels with the help of statistical method. The combination of texture of red channel, green channel and blue channel we create a feature vector for all images of the database. This proposed method is performed on the Corel dataset. Experimental results are shows the average accuracy, average precision rate and average recall rate. That is better than other existing methods like WDH (wavelet decomposed color histogram) and CCV (color coherence vector). In our proposed method we extract the color and texture features of the original image but if we extract the shape feature of the image after extraction of the color channel like red channel, green channel and blue channel. After then we perform the texture analysis and obtain the feature vector of color, shape and texture then we get the better results from the current results. And we can perform this method on the different and large datasets.

\section{REFERENCES}

[1] R. Jain. "Visual Information Management Systems." Proc. US NSF Workshop, ed., 1992.

[2] Arnold W.M. Smeulders, S, Marcel Worring, Simone Santini, Amarnath Gupta, and Ramesh Jain. ContentBased Image Retrieval at the End of the Early Years. IEEE TRANSACTIONS ON PATTERN ANALYSIS AND MACHINE INTELLIGENCE, VOL. 22, NO. 12, DECEMBER 2000.

[3] Mona Mahrous Mohammeda, Amr Badrb, M.B. Abdelhalima. Image classification and retrieval using optimized Pulse-Coupled Neural Network. doi:10.1016/j.eswa.2015.02.019.

[4] Sourabh Shrivastava, Satish Kumar Singh, Dhara Singh Hooda, "Statistical Texture and Normalized Discrete Cosine Transform based Automatic Soya Plant Foliar Infection Cataloguing," British Journal of Mathematics and Computer Sciences, vol. 4, no. 20, pp. 2901-2916, 2014.

[5] Pedro H. Bugatti, Daniel S. Kaster, Marcelo PoncianoSilva, Caetano Traina Jr., Paulo M. Azevedo-Marques, Agma J.M. Traina. PRoSPer: Perceptual similarity queries in medical CBIR systems through user profiles Original Research ArticleComputers in Biology and Medicine, Volume 45, 1 February 2014, Pages 8-19.

[6] Manisha Verma, Balasubramanian Raman, Subrahmanyam murala. "Local extrema co-occurrence pattern for color and texture image retrieval." Volume 165, Pages 255-269, 1 October 2015.

[7] Meng Jian, Cheolkon Jung, Yanbo Shen, Juan Liu. "Interactive image retrieval using constraints." Volume 161, Pages 210-219, 5 August 2015.

[8] Reshma Khemchandani, Pooja Saigal. "Color image classification and retrieval through ternary decision structure based multi-category TWSVM." Volume 165, Pages 444-455, 1 October 2015.

[9] P. Vijaya Bhaskar reddy, A. Rama Mohan Reddy. "Content based image indexing and retrieval using directional local extrema and magnitude patterns." Volume 68, Issue 7, Pages 637-643, July 2014.

[10] M. Yasmin M. Sharif. “An Efficient Content Based Image Retrieval using EI Classification and Color Features." Volume 12, Issue 5, Pages 877-885, October 2014.

[11] Mohsen Sardari Zarchi, Amirhasan Monadjemi. "A semantic model for general purpose content-based image retrieval systems." Volume 40, Issue 7, Pages 2062 2071, October 2014. 
[12] Ghanshyam Raghuwanshi, Vipin Tyagi. "Texture image retrieval using adaptive tetrolet transforms." Volume 132, Issue 4, Pages 50-57, January 2016.

[13] Santosh Kumar Vipparthi, Subrahmanyam Murala, Shyam Krishna Nagar. "Dual directional multi-motif XOR patterns: A new feature descriptor for image indexing and retrieval." Volume 126, Issues 15-16, Pages 1467-1473, August 2015.
[14] Hong-Ying Yang, Na Xu, Wei-Yi Li. "Color image representation using invariant exponent moments "Volume 46, Pages 273-287, August 2015.

[15] Michael B. Martin and Amy E. Bell, "New Image Compression Techniques using Multi wavelets and Multi wavelet Packets", IEEE Transactions on image processing, Vol. 10, No. 4, April 2001.

[16] Hiremath P. S, Shivashankar. S. "Wavelet based features for texture classification", GVIP Journal, Vol.6, Issue 3, pp 55-58, December 2006 